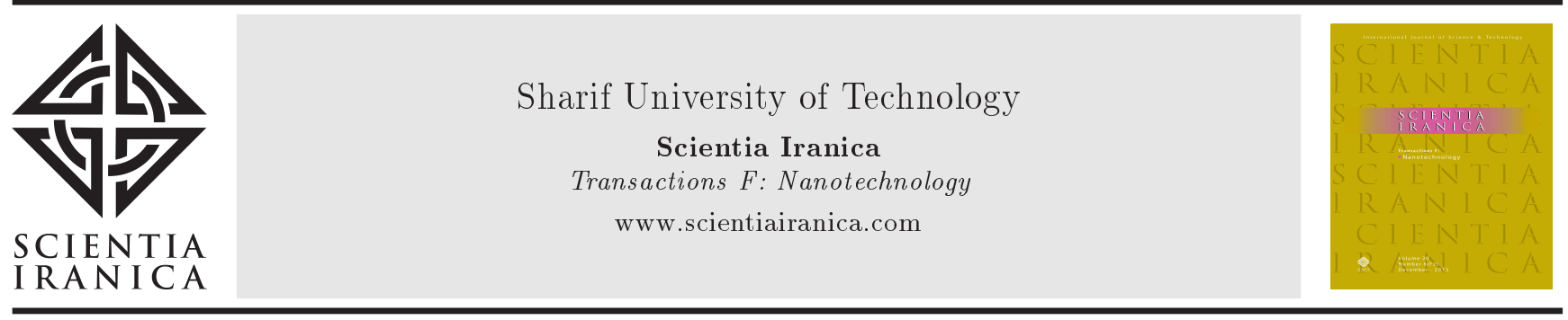

\title{
Terahertz plasmonic switch based on periodic array of graphene/silicon
}

\author{
M. Derakhshi and D. Fathi* \\ School of Electrical and Computer Engineering, Tarbiat Modares University (TMU), Tehran, P.O. Box 14115-194, Iran.
}

Received 8 August 2016; received in revised form 7 October 2016; accepted 4 March 2017

\author{
KEYWORDS \\ Plasmonic waveguide; \\ Switch; \\ Graphene; \\ Anisotropic; \\ Terahertz.
}

\begin{abstract}
We present a plasmonic switch based on anisotropic structure consisting of graphene and silicon layers, such as one-dimensional photonic crystal. The plasmonic wave in this switch is propagated inside $\mathrm{MgF}_{2}$ core surrounded by two periodic arrays of graphene/silicon. By changing the chemical potential of graphene leading to the change of permittivity of anisotropic array, we can adjust the propagation length of plasmonic wave. The obtained results show that, at the frequency of $30 \mathrm{THz}$ with adjusting the chemical potential of graphene, the chemical potential of $0.4 \mathrm{eV}$ and $0.2 \mathrm{eV}$ can be obtained for the $\mathrm{ON}$ and $\mathrm{OFF}$ states, respectively. In addition, the $\mathrm{ON} / \mathrm{OFF}$ ratio is about 24.

(C) 2017 Sharif University of Technology. All rights reserved.
\end{abstract}

\section{Introduction}

Recent studies have been focused on integrated plasmonic devices for their applications in optical circuits in order to be used in telecommunication and data transmission, causing the development of plasmonic devices in recent years [1]. The study on $\mathrm{THz}$ range for use in the areas of semiconductors, medicine, defense, telecommunication, sensors and spectroscopy has been an interest of researchers [2-4]. The studies were carried out on the propagation of plasmonic wave in a two-dimensional graphene $[5,6]$. The grapheme-based plasmonic nanostructures surrounding the plasmonic wave in small sizes, which are adjustable with the chemical potential and gate voltage, can be used to fabricate the integrated plasmonic devices in the range of infrared to terahertz [7-9]. The proposed structure for designing the plasmonic switch and studying the effects of various physical phenomena on the switch

\footnotetext{
*. Corresponding author. Tel.: +98 2182884973 ;

Fax: +982182884325

E-mail address: d.fathi@modares.ac.ir (D. Fathi)
}

efficiency has been reviewed [10]. The switching performance of graphene plasmonic waveguides for phase and extinction modulations has been studied and shown that are highly configurable with the change of Fermi levels of graphene waveguide [11]. In addition, a non-resonant all-optical nonlinear graphene plasmonic waveguide has been investigated, which has a high switching speed with an extinction ratio of $18.14 \mathrm{~dB}$ working at the $\mathrm{THz}$ frequency based on photo-induced absorption change [12]. Graphene plasmonic response in the Pauli blocking regime can cause the spectral shifts of Fano resonances that can enable the reflective modulation by nearly an order of magnitude [13]. In addition, it has been shown that the interaction between graphene and light can be utilized to extract the optical parameters of graphene [13]. When the imaginary part of conductivity of graphene becomes positive by applying a gate voltage or changing the chemical potential, graphene acts like a thin layer of metal and can support the plasmonic waves [14]. Graphene is a two-dimensional material of carbon atoms bonded to each other in a honeycomb structure. Doped graphene with smaller frequencies compared to the frequencies of optical phonons is capable of carrying the low loss plasmons and high loss levels of plasmons caused by 
the transitions between bands, which can be eliminated by high doping concentrations $[15,16]$. Recent studies have shown that the fabrication of graphenebased plasmonic devices has benefits in some ways compared to gold and silver due to the adjustability by changing the impurities and gate voltage [14]. The plasmonic devices are capable to propagate or block the plasmonic wave. In this regard, the plasmonic switches based on graphene have been investigated at different frequency ranges $[17,18]$. In addition, the effect of cover anisotropy on the propagation of plasmonic wave in plasmonic waveguides has been studied $[19,20]$.

\section{Materials and method}

The proposed plasmonic switch can be observed in Figure 1. Two anisotropic coverages as one-dimensional graphene/silicon photonic crystal are placed on both

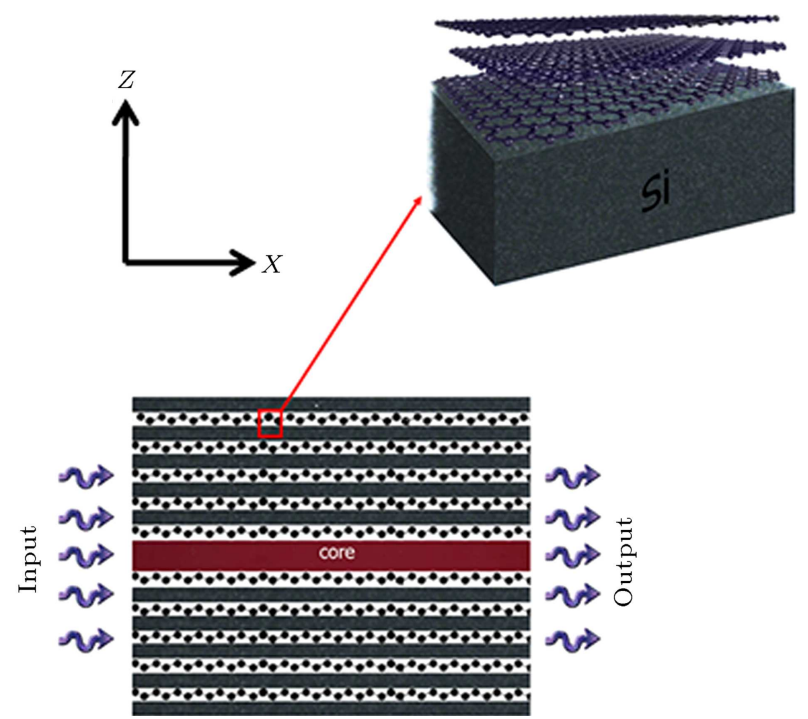

Figure 1. The structure of the proposed plasmonic switch based on periodic array of graphene/silicon with the unit cell of anisotropic clad.

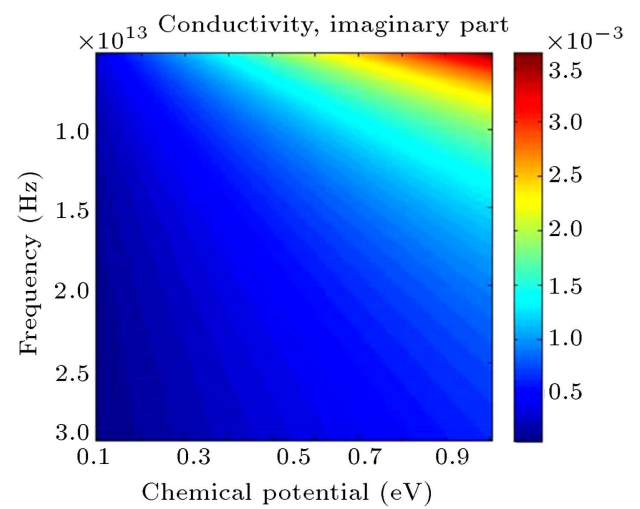

(a) sides of a dielectric material with the permittivity of $\varepsilon_{d}=1.88$.

Since the one-dimensional photonic crystal is an anisotropic material, permittivities $\varepsilon_{x}$ and $\varepsilon_{z}$ are parallel with and perpendicular to the direction of radiation, respectively. For the double-layer structure, the permittivities parallel with and perpendicular to the direction of radiation can be derived from [21].

$$
\varepsilon_{x}=\varepsilon_{g} f+\varepsilon_{s i}(1-f), \quad \varepsilon_{z}=\frac{1}{\frac{f}{\varepsilon_{g}}}+\frac{(1-f)}{\varepsilon_{s i}},
$$

where $f$ is the filling factor of graphene in the anisotropic graphene/silicon structure; $\varepsilon_{s i}$ is the permittivity of silicon; $\sigma_{g}$ and $\varepsilon_{g}$ are the conductivity and permittivity of graphene, respectively, which can be calculated using the Kubo formula as follows [14,22]:

$$
\begin{aligned}
\sigma_{g} & =i \frac{e^{2} k_{B} T}{\pi \hbar^{2}(\omega+j 2 \Gamma)}\left[\frac{\mu}{k_{B} T}+2 \ln \left(e^{-\frac{\mu}{k_{B} T}}+1\right)\right] \\
\varepsilon_{g} & =1+j\left(\frac{\sigma_{g} \eta_{0}}{k_{0} \Delta}\right)
\end{aligned}
$$

where $k_{B}, \omega, j, e, T, \hbar$ are the Boltzmann constant, angular frequency, imaginary unit, charge of electron, absolute temperature, and reduced Planck's constant, respectively. Also, $\eta_{0}=377 \Omega$ is the impedance of air, $\Delta$ is the effective thickness of graphene (is considered $0.3 \mathrm{~nm}$ in here), $k_{0}=\omega / c$ is the vacuum wave vector where $c$ is the speed of light, and $\mu$ is the chemical potential of graphene, which can be changed by the applied bias voltage.

$I_{m}\left(\sigma_{g}\right)$ can get positive or negative values, where graphene acts as a thin metallic layer for positive values, so it can support the surface plasmon polariton waves. Figure 2 shows the real and imaginary parts of the conductivity of graphene. As this figure shows, $I_{m}\left(\sigma_{g}\right)>0$ for almost all ranges of frequency and

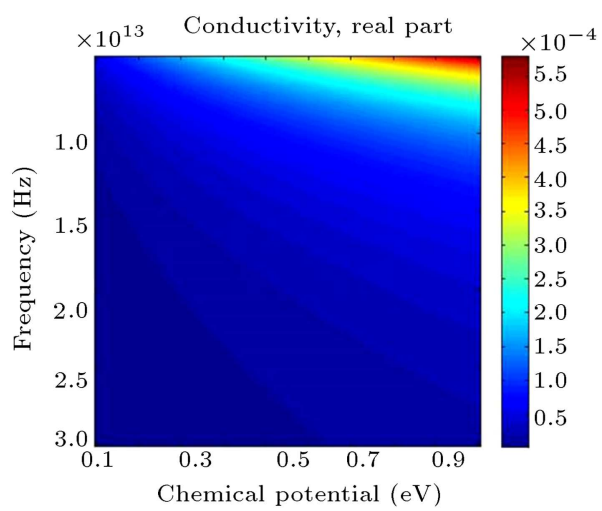

(b)

Figure 2. Profile of the conductivity of graphene versus the frequency and chemical potential variations: (a) The imaginary part, and (b) the real part. 


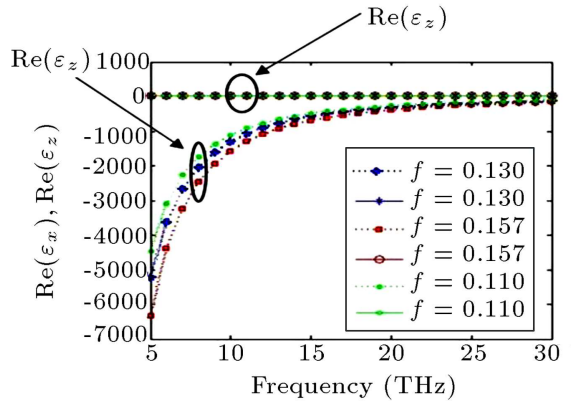

(a)

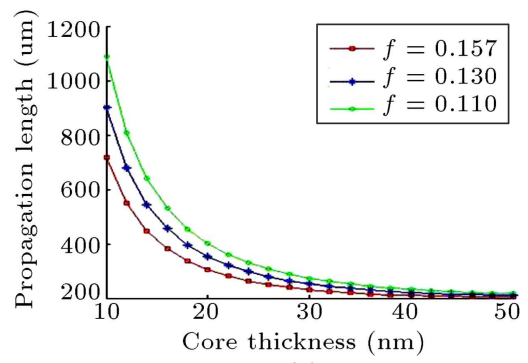

(c)

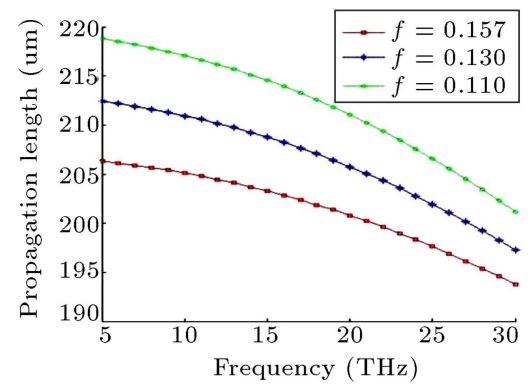

(b)

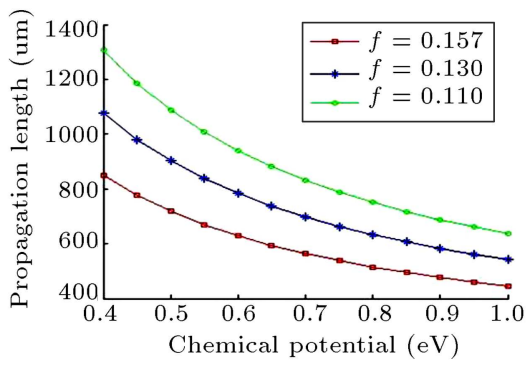

(d)

Figure 3. (a) The real parts of permittivities of clad versus the frequency. (b) The propagation length versus the frequency. (c) The propagation length versus the core thickness. (d) The propagation length versus the chemical potential for different values of filling ratio in the clad.

chemical potential; so, graphene can support the TM surface plasmon polariton waves.

The interband and intraband transitions affect the conductivity of graphene. When the chemical potential increases, the intraband transition is dominant; so, the conductivity is entirely due to the intraband transition. In the proposed switch structure of Figure 1 , it is assumed that the core has been occupied between $0<z<d$ space. The plasmonic wave with the TM polarization propagates at both common boundaries of the core and anisotropic coverage. The plasmonic wave has two electrical components, $E_{x}$ and $E_{z}$, and only one magnetic component, $H_{y}=H(x, z)$. The magnetic field inside the core is formed by the superposition of two exponential terms as:

$$
H_{y 1}(x, z)=A \exp \left(i k x+\kappa_{1} z\right)+B \exp \left(i k x-\kappa_{1} z\right),
$$

in the down and up clad-core interfaces:

$$
\begin{aligned}
& H_{y 2}(x, z)=C \exp \left[i k x+\kappa_{2} z\right], \\
& H_{y 3}(x, z)=d \exp \left[i k x-\kappa_{2}(z-d)\right],
\end{aligned}
$$

respectively, where $A, B, C$, and $d$ are constants; $k$ is the surface plasmon wave vector; $\kappa_{1}$ and $\kappa_{2}$ are the reverse penetration depths in the core and clad, respectively, defined as follows:

$$
\kappa_{1}=\sqrt{k^{2}-\varepsilon_{d} \omega^{2} / c^{2}}, \quad \kappa_{2}=\sqrt{\varepsilon_{x}\left(\frac{k^{2}}{\varepsilon_{z}}-\frac{\omega^{2}}{c^{2}}\right)} .
$$

In order to satisfy the continuity conditions of magnetic fields at the clad-core interface and considering the electric field, the dispersion relation can be obtained using [19]:

$$
\frac{\kappa_{2} \varepsilon_{d}}{\kappa_{1} \varepsilon_{x}}=-\tanh \left(\frac{\kappa_{1} d}{2}\right) .
$$

Note that $k=k^{\prime}+i k^{\prime \prime}$ is complex with the real and imaginary parts. According to the imaginary part of $k$, one can calculate the propagation length of surface plasmon polariton inside the waveguide using $L=1 /\left(2 k^{\prime \prime}\right)[19]$ :

$$
\begin{aligned}
L(\omega)= & \frac{k}{2 \varepsilon_{d}} \frac{1}{\kappa_{2}^{2}}\left[\frac{\varepsilon_{x}}{\varepsilon_{z}}-\frac{\kappa_{2}^{2}}{\kappa_{1}^{2}}+d \frac{\kappa_{2}}{2}\left(\frac{\left|\varepsilon_{d}\right|}{\varepsilon_{x}} \frac{\kappa_{2}^{2}}{\kappa_{1}^{2}}-\frac{\varepsilon_{x}}{\left|\varepsilon_{d}\right|}\right)\right] \\
& \times\left[\frac{1}{\left|\varepsilon_{d}\right|}-\frac{\omega^{2}}{2 c^{2} \kappa_{1}^{2}}+\frac{\omega^{2}}{4 c^{2}} \frac{d}{\kappa_{2}}\left(\frac{\left|\varepsilon_{d}\right|}{\varepsilon_{x}} \frac{\kappa_{2}^{2}}{\kappa_{1}^{2}}-\frac{\varepsilon_{x}}{\left|\varepsilon_{d}\right|}\right)\right]^{-1} .
\end{aligned}
$$

Changing the chemical potential of graphene and the physical structure of waveguide, we can control the length of plasmonic wave propagation inside the waveguide. Figure 3(a) shows the variations of $\operatorname{Re}\left(\varepsilon_{x}\right)$ and $\operatorname{Re}\left(\varepsilon_{z}\right)$ versus the frequency in the terahertz range, with the applied chemical potential $\mu=0.5 \mathrm{eV}$ for three different filling ratios $f=0.11,0.13,0.157$. As shown in this figure, $\operatorname{Re}\left(\varepsilon_{x}\right)$ is negative for all frequencies, so the clad behaves like a metal. When filling ratio $f$ is changed, the clad permittivity will change; consequently, we can adjust the propagation length of plasmonic wave.

Figure 3(b) shows the variation of propagation length versus the frequency. This property enables us 
to build devices, such as switches and gates, which can pass waves in a certain range of frequency and cannot pass in other ranges.

Figure 3(c) shows the effect of physical structure on the propagation length. Changing the physical structures, such as the filling ratio and core thickness, will cause the flexibility of waveguide.

In Figure 3(d), we can see the flexibility of waveguide by changing the graphene chemical potential. The main advantage of using graphene in the structure is the flexibility of waveguide behavior without modifying the physical structure and frequency.

The curves of Figure 3 have been plotted for the case of using three-layered graphene. Increasing the number of graphene layers causes the propagation length of plasmonic wave to increase. A switching behavior could be observed by adjusting the chemical potential of graphene. This means that, with a high value of chemical potential, we have a high propagation length of plasmon polariton wave, whereas, with a low value of chemical potential, the propagation length will be very low or not at all [23].

\section{Results and discussion}

The structure of electro-optic switch shown in Figure 1 has a length of $5 \mu \mathrm{m}$. In addition, the thickness of core inside which the wave propagates is $50 \mathrm{~nm}$, and the filling factor is $f=0.017$ (the effective thickness of graphene is proportional to the thickness of a unit cell of anisotropic coating). The core is $\mathrm{MgF}_{2}$ with the permittivity of $\varepsilon_{d}=1.88$. On the other hand, graphene has one layer in the switch structure. The simulation has been performed using a specific port on the plasmonic waves inside the switch. Changing the chemical potential in the graphene layer, the propagation length would be adjustable. Figure 4 shows the variation of propagation length versus the chemical potential in

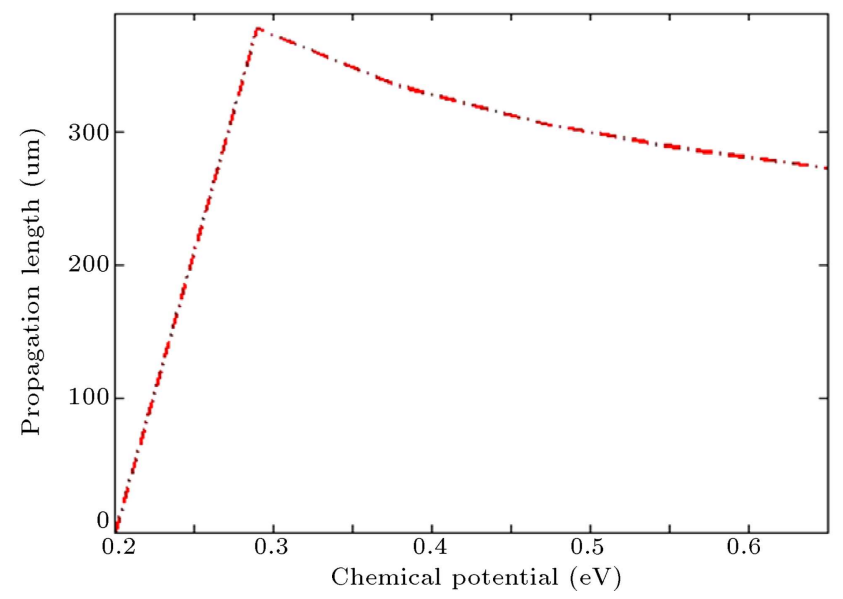

Figure 4. The propagation length versus the chemical potential at the frequency of $30 \mathrm{THz}$.

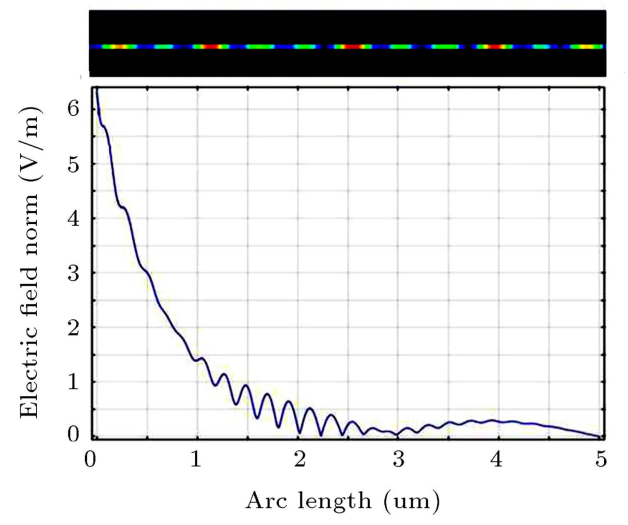

(a)

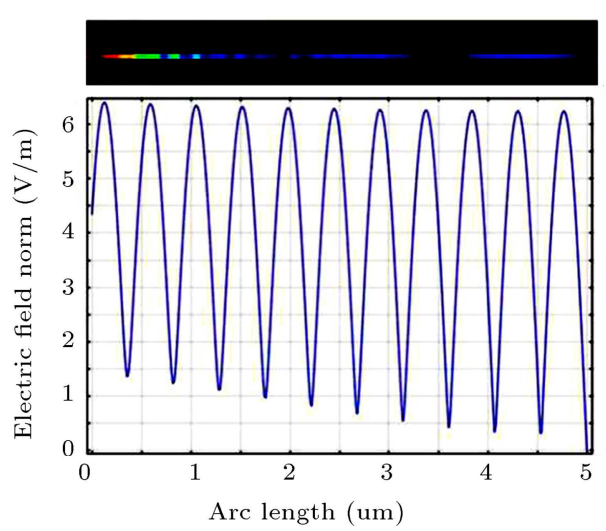

(b)

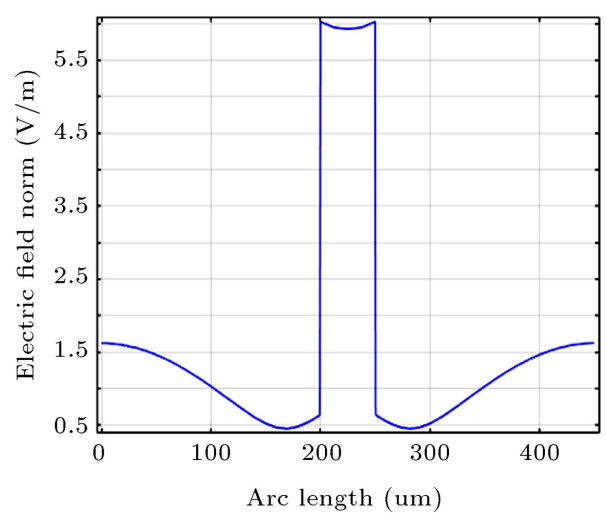

(c)

Figure 5. Profile of the electric field distribution for (a) the OFF state, (b) the ON state, and (c) the vertical direction.

the graphene layer between $0.2 \mathrm{eV}<\mu<0.65 \mathrm{eV}$ at $30 \mathrm{THz}$ frequency. According to this figure, for small values of the chemical potential, less than $0.3 \mathrm{eV}$, there is a very low propagation length, whereas high values of chemical potential, more than $0.3 \mathrm{eV}$, give rise to high values of propagation length. Therefore, the chemical potential of graphene for the OFF and ON states of switch has been assumed equal to $0.2 \mathrm{eV}$ and $0.4 \mathrm{eV}$, respectively.

Figure 5 shows the profile of electric field in the 
vertical and horizontal directions of waveguide. For the chemical potential $\mu=0.2 \mathrm{eV}$, the plasmonic wave loss inside the waveguide is high, whereas, with the chemical potential $\mu=0.4 \mathrm{eV}$, the plasmonic wave passes with very low loss. Figure 5 (c) shows the profile of electric field in the vertical direction. As can be observed, the electric field is concentrated inside the core; also, the maximum intensity therein occurs at the clad-core interface.

If we define the transmission rate of plasmonic wave for the switch structure as the ratio of output electric field to the input electric field:

$$
\text { Transmission }=E_{\text {output }} / E_{\text {input }}
$$

as shown in Figure 5, for the OFF state, approximately $4 \%$ of input plasmonic wave reaches the output, whereas, for the ON state, almost $96 \%$ of plasmonic wave will be transferred to the output. This means that we have achieved the transmission rate of plasmonic wave equal to $\mathrm{ON} / \mathrm{OFF}=24$.

\section{Conclusions}

In this paper, a plasmonic waveguide based on an array of graphene/silicon in the terahertz frequency range, acting as a plasmonic switch, was presented. It was observed that, in this waveguide, we could adjust the propagation length by changing the physical structure of clad and the chemical potential of graphene. Therefore, hereby, we are able to design a new plasmonic switch. In addition, we observed that, at the frequency of $30 \mathrm{THz}$ with the chemical potential of graphene equal to $0.2 \mathrm{eV}$, the propagation length of plasmonic wave is low, whereas, for the chemical potential of graphene equal to $0.4 \mathrm{eV}$, we have a high ratio for the propagation length of plasmonic wave with respect to the switch length. As a final result, we are able to design a plasmonic switch with the transmission rate of plasmonic wave equal to $\mathrm{ON} / \mathrm{OFF}=24$.

\section{References}

1. Berweger, S., Atkin, J.M., Xu, X.G., Olmon, R.L. and Raschke, M.B. "Femtosecond nanofocusing with full optical waveform control", Nano letters, 11(10), pp. 4309-4313 (2011).

2. Brucherseifer, M., Nagel, M., Bolivar, P.H., Kurz, H., Bosserhoff, A. and Büttner, R. "Label-free probing of the binding state of DNA by time-domain terahertz sensing", Applied Physics Letters, 77(24), pp. 40494051 (2000).

3. Kleine-Ostmann, T. and Nagatsuma, T. "A review on terahertz communications research", Journal of Infrared, Millimeter, and Terahertz Waves, 32(2), pp. 143-171 (2011).
4. Chen, H.T., Kersting, R. and Cho, G.C. "Terahertz imaging with nanometer resolution", Applied Physics Letters, 83(15), pp. 3009-3011 (2003).

5. Ferreira, A., Peres, N.M.R. and Neto, A.C. "Confined magneto-optical waves in grapheme", Physical Review $B, \mathbf{8 5}(20)$, p. 205426 (2012).

6. Gómez-Díaz, J.S. and Perruisseau-Carrier, J. "Propagation of hybrid transverse magnetic-transverse electric plasmons on magnetically biased graphene sheets", Journal of Applied Physics, 112(12), p. 124906 (2012).

7. Grigorenko, A.N., Polini, M. and Novoselov, K.S. "Graphene plasmonics", Nature Photonics, 6(11), pp. 749-758 (2012).

8. Gan, C.H. "Analysis of surface plasmon excitation at terahertz frequencies with highly doped graphene sheets via attenuated total reflection", Applied Physics Letters, 101(11), p. 111609 (2012).

9. Yan, H., Li, X., Chandra, B., Tulevski, G., Wu, Y., Freitag, M., Zhu, W., Avouris, P. and Xia, F. "Tunable infrared plasmonic devices using graphene/insulator stacks", Nature Nanotechnology, 7(5), pp. 330-334 (2012).

10. Emboras, A., Hoessbacher, C., Haffner, C., Heni, W., Koch, U., Ma, P. Fedoryshyn, Y., Niegemann, J., Hafner, C. and Leuthold, J. "Electrically controlled plasmonic switches and modulators", IEEE Journal of Selected Topics in Quantum Electronics, 21(4), pp. 276-283 (2015).

11. Ooi, K.J., Cheng, J.L., Sipe, J.E., Ang, L.K. and Tan, D.T. "Ultrafast, broadband, and configurable midinfrared all-optical switching in nonlinear graphene plasmonic waveguides", APL Photonics, 1(4), p. 046101 (2016).

12. Li, J., Tao, J., Chen, Z.H. and Huang, X.G. "Alloptical controlling based on nonlinear graphene plasmonic waveguides", Optics Express, 24(19), pp. 2216922176 (2016).

13. Dabidian, N., Kholmanov, I., Khanikaev, A.B., Tatar, K., Trendafilov, S., Mousavi, S.H., Magnuson, C., Ruoff, R.S. and Shvets, G. "Electrical switching of infrared light using graphene integration with plasmonic Fano resonant metasurfaces", Acs Photonics, 2(2), pp. 216-227 (2015).

14. Vakil, A. and Engheta, N. "Transformation optics using graphene", Science, 332(6035), pp. 1291-1294 (2011).

15. Ju, L., Geng, B., Horng, J., Girit, C., Martin, M., Hao, Z., Bechtel, H.A., Liang, X., Zettl, A., Shen, Y.R. and Wang, F. "Graphene plasmonics for tunable terahertz metamaterials", Nature Nanotechnology, 6(10), pp. 630-634 (2011).

16. Liu, M., Yin, X., Ulin-Avila, E., Geng, B., Zentgraf, T., Ju, L., Wang, F. and Zhang, X. "A graphene-based broadband optical modulator", Nature, 474(7349), pp. 64-67 (2011). 
17. Standley, B., Bao, W., Zhang, H., Bruck, J., Lau, C.N. and Bockrath, M. "Graphene-based atomic-scale switches", Nano Letters, 8(10), pp. 3345-3349 (2008).

18. Bludov, Y.V., Vasilevskiy, M.I. and Peres, N.M.R. "Mechanism for graphene-based optoelectronic switches by tuning surface plasmon-polaritons in monolayer grapheme", EPL (Europhysics Letters), 92(6), p. 68001 (2011).

19. Krokhin, A.A. "Long-range surface plasmons in dielectric-metal-dielectric structure with highly anisotropic substrates", Physical Review B, 81(8), p. 085426 (2010).

20. Ishii, S., Shalaginov, M.Y., Babicheva, V.E., Boltasseva, A. and Kildishev, A.V. "Plasmonic waveguides cladded by hyperbolic metamaterial", Optics Letters, 39(16), pp. 4663-4666 (2014).

21. Wood, B., Pendry, J.B. and Tsai, D.P. "Directed subwavelength imaging using a layered metal-dielectric system", Physical Review B, 74(11), p. 115116 (2006).

22. Xu, C., Jin, Y., Yang, L., Yang, J. and Jiang, X. "Characteristics of electro-refractive modulating based on graphene-oxide-silicon waveguide", Optics Express, 20(20), pp. 22398-22405 (2012).

23. Derakhshi, M. and Fathi, D. "Graphene-based surface plasmon waveguide with the anisotropic clad", 24th Iranian Conference on Electrical Engineering (ICEE) (2016).

\section{Biographies}

Mahmoud Derakhshi received his BSc degree in the field of Electronics from Shahid Chamran University, Ahwaz, Iran in 2012, and the MSc degree in the field of Optoelectronics from Tarbiat Modares University (TMU), Tehran, Iran in 2015. His research interests include graphene plasmonics; plasmonic waveguides; plasmonic gates and switches.

Davood Fathi received the BSc degree in the field of Electronic Engineering from Amirkabir University of Technology, Tehran, Iran in 1990, and the MSc degree in the field of Biomedical Engineering from Sharif University of Technology, Tehran, Iran in 1994. After a couple of years working in industry, he worked toward the PhD degree between 2006-2009 in the field of nanoelectronics with the Nanoelectronic Center of Excellence, Thin Film and Photonics Research Laboratory, School of Electrical and Computer Engineering, University of Tehran, Tehran, Iran. Dr. Fathi has joined from 2010 as a member of faculty to the School of Electrical and Computer Engineering, Tarbiat Modares University (TMU), Tehran, Iran. His current research interests include nanoelectronics; nanophotonics and optoelectronics; solar cells; and quantum transport. He is also author or coauthor of more than 45 journal and conference papers in various fields of nanoelectronics and nanophotonics. 